\title{
Ansia e depressione nella popolazione generale
}

\author{
CARLO FARAVELLI, SABRINA PATERNITI, ALEJANDRA TABERNA
}

\section{INTRODUZIONE}

Negli anni '50-'60 l'epidemiologia psichiatrica ricevette un forte impulso, in seguito alla dimostrazione dell'alta frequenza dei disturbi psichiatrici, sia a livello di comunità che tra i pazienti afferenti ai medici di base (Shepherd et al., 1966; Goldberg \& Blackwell, 1970), tra i quali si riscontra una prevalenza di morbilità psichiatrica che si aggira tra il $25 \%$ e il $35 \%$ (Skuse \& Williams, 1984).

Con l'adozione dei criteri diagnostici operazionali (in primis Research Diagnostic Criteria e criteri del DSM-III) si ebbe il cosiddetto «Rinascimento della epidemiologia", che vide il fiorire di diversi studi di popolazione su larga scala: lo studio di New Haven del 1975-76 (Weissman et al., 1978), la National Survey del 1979 (Uhlenhuth et al., 1983), e poi il più noto studio ECA (Epidemiologic Catchment Area), che coinvolge alcune città americane (Regier et al., 1984), a cui seguirono varie altre ricerche in varie parti del mondo. Caratteristiche di studio peculiari furono adottate a Zurigo e a Monaco, dove fu adottata una metodologia di indagine epidemiologica prospettica. Ai primi anni ' 90 , infine, risalgono le prime collaborazioni internazionali, che hanno permesso l'esecuzione di studi epidemiologici transculturali (Cross-National Collaborative Study).

Elemento comune di tutti questi studi è stato quello di ribadire e amplificare ulteriormente il dato, già segnalato negli anni ' 50 , di una altissima prevalenza delle patologie legate ad ansia e depressione.

Indirizzo per la corrispondenza: Professor C. Faravelli, Dipartimento di Scienze Neurologiche e Psichiatriche, viale Morgagni 85, 50134 Firenze.

Fax (+ 39) 055 - 4222.599

\section{TASSI DI PREVALENZA}

\section{Disturbi dell'umore}

La tabella I mostra i tassi di prevalenza dei disturbi depressivi «pre-DSM-III», dove si possono rilevare le consistenti differenze tra gli studi che hanno utilizzato registri medici rispetto a quelli che si sono basati su indagini di comunità, sostanziando così il concetto di iceberg della depressione.

Le tabelle II, III e IV riassumono i valori riportati dagli studi di popolazione che hanno utilizzato criteri diagnostici operazionali. Considerando la variabilità intrinseca agli studi epidemiologici si può notare una sostanziale omogeneità di risultati, almeno nei Paesi di cultura occidentale (Canada, Nuova Zelanda, Monaco, Parigi, Firenze), con valori medi di prevalenza lievemente più bassi negli Stati Uniti. Rilevanti differenze si notano invece tra luoghi di cultura differente: valori massimi a Beirut, dove lo studio è stato effettuato durante il periodo bellico, e minimi a Taiwan. Sull'origine di tali differenze si possono fare diverse ipotesi, soprattutto relative ai diversi substrati socio-storico-culturali dei Paesi, ma studi più approfonditi sono necessari per stabilire con più sicurezza la validità delle stesse.

Gli altissimi tassi di prevalenza dei disturbi dell'umore registrati negli ultimi anni inducono a prendere in considerazione alcuni problemi nosografici. Nonostante che le recenti classificazioni stabiliscano criteri più rigorosi nella definizione della depressione maggiore, il dubbio sollevato da molti clinici è che i criteri di inclusione rimangano troppo ampi, tali da comprendere nella definizione di disturbo casi che rappresentano invece solo un disagio esistenziale.

D'altra parte, lo studio dell'utilizzazione delle risorse sanitarie da parte dei soggetti affetti da depressione maggiore (vedi oltre) fa pensare che almeno questa forma costituisca una malattia «reale» e non solo una forma aspecifica di disagio. 
C. Faravelli et al.

Tabella I. - Prevalenza \% della depressione unipolare prima del 1980 (diagnosi non basata su criteri operazionali).

\begin{tabular}{llll}
\hline Autore & Area geografica & Periodo & Prevalenza \% \\
\hline Fonte: registri medici & & & 0.1 \\
Lemkau et al., 1941 & Baltimora, USA & 1 anno & 0.4 \\
Lin, 1953 & Taiwan & 6 mesi & 0.9 \\
Eaton \& Weil, 1955 & Utteriti, USA & 1 anno & 0.3 \\
Yoo, 1961 & Sud Corea & 5 anni & 0.3 \\
Watts et al., 1964 & Galles, Scozia & 1 anno & 0.3 \\
Wing et al., 1967 & Baltimora, USA & 1 anno & 0.9 \\
Mazer, 1967 & Massachussets, USA & 3 mesi & 1.5 \\
Bebbington, 1978 & Birmingham, UK & 1 anno & 1.0 \\
Nielsen \& Nielsen, 1979 & Danimarca & punto & \\
Fonte: popolazione generale & & & 3.9 \\
Sorensen \& Stromgren, 1961 & Danimarca & punto & 7.8 (f) \\
Hallstrom, 1973 & Svezia & punto & 8.4 (f) \\
Brown \& Harris, 1978 & Ebridi & 1 anno & 6.5 \\
Brown \& Harris, 1978 & Londra, UK & 1 anno & 10.8 \\
Weissman \& Myers, 1978 & New Haven, USA & 1 anno & \\
Duncan-Jones \& Henderson, 1978 & Canberra, Australia & punto & \\
(f) $=$ campione di solo femmine & & & \\
\hline
\end{tabular}

\section{Disturbi d'ansia}

Come riportato da Marks \& Lader (1973), gli studi condotti tra il 1943 e il 1966 trovarono una buona concordanza nei risultati relativi alla prevalenza-punto (prevalenza del disturbo in un determinato momento) degli «stati d'ansia», che risultò variare tra il $2.0 \%$ e il $4.7 \%$.

Studi successivi, tuttavia, che presero in considerazione criteri diagnostici più articolati, hanno messo in evidenza una buona concordanza per alcune diagnosi ben definite (Disturbo di panico, Disturbo ossessivo-compulsivo), ma una notevole variabilità nei tassi di altre diagnosi (Fobie, Disturbo d'ansia generalizzata [DAG]) (tabella V).

Ci si può chiedere in che misura tali differenze rispecchino diversità nella definizione di tali patologie e nella metodologia di raccolta dei casi e in che misura differenze reali.

I valori delle fobie, ad esempio, mostrano notevoli variazioni anche tra i risultati relativi a siti diversi appartenenti allo studio ECA.

Il DAG nelle ricerche basate sul DSM-III e sui Research Diagnostic Criteria (RDC) mostra valori molto variabili. Va considerato a questo proposito che sia il DSM-III, che è il sistema diagnostico utilizzato negli studi ECA riportati, che gli RDC usano per i disturbi d'ansia un sistema classificatorio gerarchico, che permette di fare diagnosi di DAG solo do- po l'esclusione di forme d'ansia più specifiche. La diagnosi di DAG appare così da un lato una diagnosi «in negativo», priva di una reale specificità, di cui è stata evidenziata la bassa affidabilità (DiNardo et al., 1983) e la bassa utilità (Breier et al., 1985); dall'altro lato la mancanza di specificità non consente neppure di tracciare dei limiti ben definiti con una condizione ansiosa temperamentale che sfuma nella normalità o addirittura con una sindrome distimica, il cui aspetto affettivo passi in secondo piano. Lo stesso ragionamento è valido per le fobie, dove la presenza di aspetti fobici a livello subclinico è comunissima, ma il limite con la fobia patologica è incerto.

In altre parole le maggiori divergenze si riscontrano per quei disturbi dove il confine tra normalità e patologia è sfumato e la diagnosi riposa su un certo grado di soggettività da parte dell'intervistatore.

\section{COMORBIDITÀ}

Gli studi di comorbidità esaminano la frequenza con cui patologie diverse si presentano in una stessa persona in un periodo di tempo: tutta la vita nel caso della comorbidità lifetime, la contemporanea presenza dei due disturbi nel caso della comorbidità concorrente.

Mentre abbondano gli studi clinici che esaminano la comorbidità tra ansia e depressione, solo pochi 
Ansia e depressione nella popolazione generale

Tabella II. - Prevalenza della depressione maggiore (diagnosi basata su criteri operazionali).

\begin{tabular}{|c|c|c|c|c|c|c|c|}
\hline Autore & Anno & $\begin{array}{l}\text { Area } \\
\text { geografica }\end{array}$ & $\begin{array}{l}\text { Prev. } \\
\text { punto }\end{array}$ & $\begin{array}{l}\text { Prev. } \\
6 \text { mesi }\end{array}$ & $\begin{array}{l}\text { Prev. } \\
1 \text { anno }\end{array}$ & $\begin{array}{l}\text { Prev. } \\
\text { life-time }\end{array}$ & Diagnosi \\
\hline Essen-Moller \& Hagnell, 1961 & 1947 & Scania, Svezia & & & & $4.7(f)$ & \\
\hline Murphy, 1980 & 1952 & Sterling County, Canada & 4.1 & & & & DSM-III * \\
\hline Helgason, 1961 & 1957 & Islanda & 3.8 & & & 6.0 & \\
\hline Thacore et al., 1975 & $1965 / 6$ & Northern India & 1.2 & & & & \\
\hline Sorensen \& Stroemgren, 1961 & 1970 & Samso Island, Danimarca & 3.9 & & & & ICD-8 \\
\hline Bash \& Bash-Liechti, 1964 & 1970 & Shiraz, Iran & 1.1 & & & & \\
\hline Blazer \& Williams, 1980 & 1972 & North Carolina, USA & 3.7 & & & & DSM-III * \\
\hline Wing et al., 1978 & $1974 / 5$ & Camberwell, UK & $9.3(f)$ & & 14.8 & & PSE/ICD-9 \\
\hline Weissman et al., 1978 & 1975 & New Haven, USA & 4.3 & & & 18.0 & RDC \\
\hline Duncan-Jones \& Henderson, & & & & & & & \\
\hline 1978 & 1976 & Canberra, Canada & 10.8 & & & & PSE/ ICD-9 \\
\hline Hallstrom, 1973 & 1980 & Gothenburg, Svezia & 6.9 & & & & DSM-III** \\
\hline \multirow{8}{*}{$\begin{array}{l}\text { Studio ECA } \\
\text { Robins et al., } 1984 \\
\text { Weissman } \text { et al., } 1988 \\
\text { Regier et al., } 1988 \\
\text { Myers et al., } 1984\end{array}$} & & USA & & & & & \\
\hline & & & & & & & \\
\hline & & & & & & & \\
\hline & & & & & & & \\
\hline & & & & & & & \\
\hline & $1980 / 1$ & New Haven, USA & 1.7 & 3.5 & & 6.7 & DSM-III \\
\hline & $1981 / 2$ & Baltimore, USA & 1.2 & 2.2 & & 3.7 & DSM-III \\
\hline & $1981 / 2$ & St. Louis, USA & 1.6 & 3.2 & & 5.5 & DSM-III \\
\hline Dean et al., 1983 & 1981 & Edimburgo, UK & $9.2(f)$ & & & & RDC \\
\hline Wittchen et al., 1992 & $1974 / 81$ & Monaco, Germania & & & 2.98 & 8.96 & DSM-III \\
\hline Hwu et al., 1989 & $1982 / 4$ & Taiwan & & & & 1.4 & DSM-III \\
\hline Bland et al., 1988a, 1988b & $1983 / 6$ & Edmonton, Canada & & 3.2 & & 8.6 & DSM-III \\
\hline Weissman et al., 1988 & 1984 & Piedmont, USA & 1.0 & 1.5 & 1.7 & 3.5 & DSM-III \\
\hline Karno et al., 1987 & 1984 & Los Angeles, USA & 1.8 & 3.1 & & 6.4 & DSM-111 \\
\hline Karno et al., 1987 & 1984 & Los Angeles, ispanici & & 3.0 & & 4.9 & DSM-III \\
\hline \multirow{2}{*}{$\begin{array}{l}\text { Canino et al., } 1987 \\
\text { Angst \& Dobler-Mikola, } \\
\text { 1985b }\end{array}$} & 1984 & Porto Rico, USA & & 3.0 & & 4.6 & DSM-III \\
\hline & 1984 & Zurigo, Svizzera & & & 4.8 & & $\begin{array}{l}\text { DSM-III, } \\
\text { RDC }\end{array}$ \\
\hline Faravelli et al., 1990 & 1984 & Firenze, Italia & 2.8 & & 6.3 & 12.0 & DSM-III \\
\hline Wells et al., 1989 & 1986 & Christchurch,Nuova Zelanda & & & & 12.6 & DSM-III \\
\hline Lepine et al., 1989 & $1987 / 8$ & Parigi , Francia & & & & 17 & DSM-III \\
\hline Karam et al., 1991 & $1988 / 9$ & Beirut, Libano & & & & 18.8 & DSM-III \\
\hline
\end{tabular}

studi hanno affrontato tale problematica sotto un profilo epidemiologico (Boyd et al., 1984; Angst \& Dobler-Mikola, 1985c; Wittchen 1988; Wittchen \& Essau, 1993).

I tassi di comorbidità tra disturbi d'ansia e depressione maggiore variano da $1.8 \%$ (prevalenza 1 anno in un campione di popolazione di $22 / 23$ anni) (Angst \& Dobler-Mikola, 1985c) a 4.4\% (prevalenza lifetime) (Wittchen \& Essau, 1993); da un ampio campione tratto dalle città coinvolte nello studio ECA, è stato calcolato che per un soggetto affetto da un episodio depressivo maggiore il rischio di avere un disturbo d'ansia è maggiore rispetto a un soggetto non affetto di una quota che varia dal $9.0 \%$ nel caso della fobia semplice al $18.8 \%$ nel caso del disturbo di panico (Boyd et al., 1984). Anche nello studio di Monaco (Wittchen \& Essau, 1993) le percentuali più alte di comorbidità sono state riscontrate a carico del disturbo di panico; tuttavia va notato che in entrambi gli studi non è stato considerato il DAG.

Wittchen \& Essau (1989) inoltre hanno conside-

Epidemiologia e Psichiatria Sociale, 3, 1, 1994 
Tabella III. - Prevalenza del disturbo bipolare.

\begin{tabular}{|c|c|c|c|c|c|c|c|}
\hline Autore & Anno & $\begin{array}{l}\text { Area } \\
\text { geografica }\end{array}$ & $\begin{array}{l}\text { Prev. } \\
\text { punto }\end{array}$ & $\begin{array}{l}\text { Prev. } \\
6 \text { mesi }\end{array}$ & $\begin{array}{l}\text { Prev. } \\
1 \text { anno }\end{array}$ & $\begin{array}{l}\text { Prev. } \\
\text { life-time }\end{array}$ & Diagnosi \\
\hline & 1957 & Islanda & & & & 0.79 & \\
\hline \multirow{6}{*}{$\begin{array}{l}\text { Weissman et al., } 1978 \\
\text { Studio ECA } \\
\text { Weissman et al., } 1988\end{array}$} & 1975 & New Haven, USA & & 0.3 & & 0.6 & RDC \\
\hline & & USA & & & & & \\
\hline & & & & & & & \\
\hline & $1980 / 7$ & New Haven, USA & 0.7 & 0.8 & 1.3 & 1.3 & DSM-III \\
\hline & $1981 / 2$ & Baltimore, USA & 0.9 & & 0.9 & & DSM-III \\
\hline & $1981 / 2$ & St. Louis, USA & 0.7 & 0.7 & 1.4 & 1.6 & DSM-III \\
\hline Weissman et al., 1988 & 1984 & Piedmont, USA & 0.4 & 0.6 & 0.6 & 1.1 & DSM-III \\
\hline Karno et al., 1987 & 1984 & Los Angeles,USA & 0.5 & 0.6 & 0.6 & 0.5 & DSM-III \\
\hline Karno et al., 1987 & 1984 & Los Angeles, ispanici & & 0.1 & & 0.3 & DSM-III \\
\hline Canino et al., 1987 & 1984 & Porto Rico, USA & & 0.3 & & 0.5 & DSM-III \\
\hline Bland et al., 1988a, b & $1983 / 6$ & Edmonton, Canada & & 0.1 & & 0.6 & DSM-III \\
\hline Faravelli et al., 1990 & 1984 & Firenze, Italia & 0.4 & & 1.3 & & DSM-III \\
\hline
\end{tabular}

Tabella IV. - Prevalenza del disturbo distimico.

\begin{tabular}{|c|c|c|c|c|c|c|c|}
\hline Autore & Anno & $\begin{array}{l}\text { Area } \\
\text { geografica }\end{array}$ & $\begin{array}{l}\text { Prev. } \\
\text { punto }\end{array}$ & $\begin{array}{l}\text { Prev. } \\
6 \text { mesi }\end{array}$ & $\begin{array}{l}\text { Prev. } \\
1 \text { anno }\end{array}$ & $\begin{array}{l}\text { Prev. } \\
\text { life-time }\end{array}$ & Diagnosi \\
\hline \multicolumn{8}{|l|}{ Weissman et al., 1988} \\
\hline & $1980 / 7$ & New Haven, USA & & 3.2 & & 3.2 & DSM-III \\
\hline & $1981 / 2$ & Baltimore, USA & & 2.1 & & 2.2 & DSM-III \\
\hline & $1981 / 2$ & St. Louis, USA & & 3.8 & & 3.9 & DSM-III \\
\hline Karno et al., 1987 & 1984 & Los Angeles, USA & & & & 4.2 & DSM-III \\
\hline Karno et al., 1987 & 1984 & Los Angeles, ispanici & & 3.4 & & 4.8 & DSM-III \\
\hline Canino et al., 1987 & 1984 & Porto Rico, USA & & & & 4.7 & DSM-III \\
\hline Bland et al., 1988 & $1983 / 6$ & Edmonton, Canada & & 3.7 & & 3.7 & DSM-III \\
\hline Faravelli $e_{t}^{t}$ al., 1990 & 1984 & Firenze, Italia & 1.0 & & 3.0 & & DSM-III \\
\hline
\end{tabular}

rato la presenza di ansia e depressione sia in un campione epidemiologico che clinico e hanno riscontrato che nel primo sono più frequenti le diagnosi «pure» di ansia o depressione, mentre nel secondo prevalgono i soggetti che sono o sono stati affetti da entrambi i disturbi. Tali risultati possono essere messi in relazione al fatto che $\mathrm{i}$ casi che presentano comorbidità tendono ad avere una prognosi peggiore, sia per ciò che riguarda la gravità della sintomatologia che della qualità della vita (Hecht \& Wittchen, 1988), ed è quindi più probabile che cerchino più frequentemente un curante. Da questa ricerca emerge inoltre che nella maggioranza dei casi il disturbo d'ansia esordisce prima del disturbo affettivo.

L'ICD-10 prevede una categoria di ansia mista a depressione (Mixed Anxiety Depressive Disorder), caratterizzata dalla presenza di sintomi di entrambi i disturbi non tale da consentire la diagnosi del singolo disturbo. La validità di tale categoria è contestata da molti nosologi: Wittchen \& Essau (1993) hanno cercato di sostanziare a livello epidemiologico tale entità diagnostica, trovando una prevalenza dello $0.8 \%$.

Un cenno infine meritano i disturbi somatoformi. Numerosi autori hanno infatti sottolineato l'associazione tra sintomi somatici e disturbi sia ansiosi (Kellner et al., 1972; Tyrer, 1976) che depressivi (Cadoret et al., 1980; Katon et al., 1982; Kellner, 1986; 1988).

Da uno studio ECA emerge (Schwartz et al., 1986) che un'alta percentuale di soggetti affetti da disturbo di somatizzazione $(77.9 \%$ ) accusava almeno 
Tabella V. - Prevalenza dei disturbi d'ansia.

\begin{tabular}{|c|c|c|c|c|c|c|c|c|c|c|}
\hline Autore & $\begin{array}{l}\text { Area } \\
\text { geografica }\end{array}$ & $\begin{array}{l}\text { Periodo } \\
\text { prev. }\end{array}$ & DAG & DP & Fobie & $\begin{array}{l}\text { AGO } \\
\pm \\
\text { PAN }\end{array}$ & $\begin{array}{l}\text { Fobia } \\
\text { SEM }\end{array}$ & $\begin{array}{l}\text { Fobia } \\
\text { SOC }\end{array}$ & DOC & Diagnosi \\
\hline Agras et al., 1969 & USA & punto & & & 7.7 & 0.6 & & & & \\
\hline Weissman et al., 1978 & New Haven, USA & punto & 2.4 & 0.4 & 1.2 & & & & 0.0 & RDC \\
\hline Bebbington et al., 1981 & Camberwell (Londra), UK & 1 mese & 7.6 & & & 0.2 & & & 0.0 & ICD-9 \\
\hline Dean et al., 1983 & Edimburgo (f), UK & 1 mese & 2.6 & 0.7 & & & & & & RDC \\
\hline Uhlenhuth et al., 1983 & USA & 1 anno & 6.4 & & & 1.2 & & & & DSM-III \\
\hline Angst \& Dobler-Mikola, 1985a & Zurigo, Svizzera & 1 anno & 5.2 & 3.1 & & 2.5 & 3.7 & 1.1 & & DSM-III \\
\hline Wittchen, 1986 & Monaco, Germania & 6 mesi & & 1.1 & & 3.6 & 4.1 & 1.8 & & DSM-III \\
\hline Wittchen, 1986 & Monaco, Germania & lifetime & & 2.4 & 5.7 & 8.0 & & 2.0 & & \\
\hline Wittchen \& von Zerssen, 1988 & Monaco, Germania & 6 mesi & & & 4.8 & & & & 0.5 & ICD-9 \\
\hline \multirow{9}{*}{$\begin{array}{l}\text { Wittchen \& von Zerssen, } 1988 \\
\text { Regier et al., } 1990\end{array}$} & Monaco, Germania & lifetime & & 6.4 & & & & 1.1 & & \\
\hline & $\begin{array}{l}\text { Studio ECA (USA) } \\
\text { St Louis, USA }\end{array}$ & 6 mesi & & 0.9 & 5.4 & 2.7 & 4.5 & 1.2 & 1.3 & DSM-III \\
\hline & St Louis, USA & lifetime & & 1.5 & 9.4 & 3.9 & 7.7 & & 1.9 & \\
\hline & Baltimora, USA & 6 mesi & & 1.0 & 13.4 & 5.8 & 11.8 & 2.2 & 2.0 & DSM-III \\
\hline & Baltimora, USA & lifetime & & 1.4 & 23.3 & & & 3.0 & & \\
\hline & New Haven, USA & 6 mesi & & 0.6 & 5.9 & 2.8 & 4.7 & & 1.4 & DSM-III \\
\hline & New Haven, USA & lifetime & & 1.4 & 7.8 & 3.5 & 6.2 & 2.6 & & \\
\hline & Los Angeles, USA & 6 mesi & & 0.9 & 6.3 & & & 0.7 & & DSM-III \\
\hline & Los Angeles, USA & lifetime & & 1.5 & 11.0 & & & 2.1 & & \\
\hline Canino et al., 1987 & Porto Rico, USA & 6 mesi & & 1.1 & 6.3 & 3.9 & 4.4 & 1.1 & 1.8 & DSM-III \\
\hline Canino et al., 1987 & Porto Rico, USA & lifetime & & 1.7 & 12.2 & 6.9 & 8.6 & 1.6 & 3.2 & \\
\hline Bland et al., 1988a, b & Edmonton, Canada & 6 mesi & & 0.7 & 5.1 & & & & & DSM-III \\
\hline Bland et al., 1988a, b & Edmonton, Canada & lifetime & & 1.2 & 8.9 & 2.9 & 7.2 & 1.7 & 3.0 & \\
\hline \multirow[t]{2}{*}{ Faravelli et al., 1989} & Firenze, Italia & punto & 2.8 & 0.3 & & 0.7 & 0.5 & 0.5 & 0.6 & DSM-III \\
\hline & & lifetime & 5.4 & 1.4 & & 0.9 & 0.6 & 1.0 & 0.7 & \\
\hline
\end{tabular}

(f) $=$ solo campione femminile

$\mathrm{DAG}=$ Disturbo d'ansia generalizzata; $\mathrm{DP}=$ Disturbo di panico; $\mathrm{AGO}=$ Agorafobia; $\mathrm{PAN}=$ attacchi di panico; FOBIA SEM = Fobia semplice; FOBIA SOC = Fobia sociale; DOC $=$ Disturbo ossessivo-compulsivo

un'altro disturbo. Le diagnosi concorrenti più frequenti sono costituite dai disturbi d'ansia $(70 \%$ : fobie) e dai disturbi affettivi (65\%: depressione maggiore). In un recente studio condotto a Firenze ( $\mathrm{Fa}$ ravelli et $a l$., in preparazione) non è emersa invece alcuna associazione significativa tra disturbi somatoformi e disturbi d'ansia o dell'umore.

\section{IMPATTO SOCIALE DI ANSIA E DEPRESSIONE}

L'impatto sociale dei disturbi d'ansia e depressivi è notevole, sia per la loro diffusione, che per diversi altri fattori. Innanzitutto a livello individuale va considerato che questi disturbi determinano un grave peggioramento della qualità di vita. È stato calcolato che lo scadimento della qualità di vita di questi soggetti è maggiore rispetto a quello dovuto a molte malattie organiche ritenute gravi, come per esempio diabete $o$ infarto cardiaco.

In secondo luogo l'impatto di tali malattie è rilevante anche a livello sociale: oltre ai costi diretti legati al trattamento, vi sono anche dei costi indiretti, legati all'assenza dal lavoro e alla perdita o riduzione della produttività, che costituisce una comune complicazione della depressione e risulta avere un costo sociale rilevante, data la diffusione della patologia, l'andamento cronico, la frequenza delle ricadute, l'impatto di questi problemi sulla famiglia (Stoudemire et al., 1986; Dew et al., 1991); tale fenomeno si fa tanto più rilevante quanto più la sua età di esordio tende a ridursi (vedi oltre) e a coinvolgere così fasce di età che sarebbero nel pieno della potenzialità produttiva (Mintz et al., 1992; Klerman \& Weissman, 1992).

Dalle ricerche epidemiologiche attuate (Shapiro et al., 1984; Faravelli et al., 1989; 1990), emerge che 
i pazienti con disturbi affettivi (in particolare depressione maggiore) e d'ansia cercano in gran parte aiuto medico; è da sottolineare, inoltre, la tendenza dei soggetti ansiosi ad assumere farmaci anche indipendentemente dalla prescrizione medica (Faravelli et al., 1989).

\section{VARIAZIONI NEL TEMPO}

Utilizzando i dati raccolti nel programma NIMHECA, Burke et al. (1991) hanno riscontrato uno spostamento dell'età di esordio della depressione verso le fasce di età più giovanili, nonché un evidente aumento della prevalenza nelle stesse fasce di età, più evidente in quelle comprese tra i 15 e i 19 anni. Da questo studio non risulta invece nessun aumento dei tassi di prevalenza dei disturbi bipolari, del disturbo di panico e delle fobie.

Più recentemente uno studio epidemiologico a cui hanno collaborato molti centri di ricerca (i 5 centri ECA negli USA, Edmonton in Canada, Porto Rico, Monaco di Baviera, Firenze, Parigi, Taiwan, Christchurch in Nuova Zelanda, Beirut) ha condotto un confronto dell'andamento temporale della depressione maggiore nei diversi Paesi considerati (Cross-National Collaborative Group, 1992). I risultati indicano una tendenza generale all'incremento dei tassi cumulativi lifetime nelle coorti più giovani; fanno eccezione i dati provenienti dai campioni ispanici di Los Angeles e Porto Rico, dove non è presente nessuna variazione. In tutti i Paesi è inoltre evidenziato un incremento dei tassi della depressione maggiore negli ultimi decenni, la cui rilevanza varia a seconda del luogo considerato.

Tale incremento non è dovuto a un artefatto legato a distorsioni della memoria tipiche del metodo retrospettivo; infatti sia le fluttuazioni nel tempo e/o di coorte in alcune località (USA, Taiwan, Libano, Firenze, Parigi) sono a breve termine.

\section{CONSIDERAZIONI FINALI}

Come abbiamo visto le patologie legate all'ansia e alla depressione costituiscono le forme di gran lunga più diffuse, rappresentando per numerosità da sole oltre i $2 / 3$ dell'intera patologia psichiatrica. Il costo sociale di tali malattie è elevatissimo sia in termini di sofferenza personale, sia in termini di impatto sul nucleo familiare, sia come impegno economico. La peculiarità di questi disturbi allo stato attuale è che essi rappresentano probabilmente i quadri dove l'intervento psichiatrico può sortire i migliori risultati. In altre parole ansia e depressione rappresentano disturbi gravi, invalidanti, frequenti e curabili. Apparirebbe quindi giustificato un impegno prioritario dei servizi diagnostico-terapeutici nei riguardi di queste forme, sia come intervento diretto, sia come sensibilizzazione dei medici e dell'opinione pubblica. Nella realtà si osserva invece che spesso le risorse dei servizi sono quasi interamente assorbite da una minoranza di pazienti psicotici cronici. La considerazione che anche ansia e depressione spesso costituiscono una patologia cronica e invalidante, con serie ripercussioni nella qualità di vita dei sofferenti, impongono impegno non solo nella terapia di tali patologie, ma anche nella ricerca e individuazione dei fattori di rischio, così da agire in senso preventivo. Anche in tale campo il ruolo dell'epidemiologia è fondamentale.

\section{BIBLIOGRAFIA}

Agras S., Sylvester D. \& Oliveau D. (1969). The epidemiology of common fears and phobia. Comprehensive Psychiatry 10, 151-156.

Angst J. \& Dobler-Mikola A. (1985a). The Zurich Study: anxiety and phobia in young adults. European Archives of Psychiatry and Neurological Sciences 235, 171-178.

Angst J. \& Dobler-Mikola A. (1985b). The Zurich Study. A prospective epidemiological study of depressive, neurotic, and psychosomatic syndromes. European Archives of Psychiatry and Neurological Sciences 234, 408-416.

Angst J. \& Dobler-Mikola A. (1985c). The Zurich Study VI. A continuum from depression to anxiety disorders. European Archives of Psychiatry and Neurological Sciences 235, 179186.

Bash K.W. \& Bash-Liechti J. (1974). Studies on the epidemiology of neuropsychiatric disorders among the population of the city of Shiraz, Iran. Social Psychiatry 9, 163-171.

Bebbington P.E. (1978). The epidemiology of depressive disorders. In Culture, Medicine and Psychiatry (ed. A.M. Kleinman), pp. 297-341. D. Reidel Putlishing Co.: Dordrecht.

Bebbington P., Hurry J., Tennant C., Sturt E. \& Wing J.K. (1981). Epidemiology of mental disorders in Camberwell. Psychological Medicine 11, 561-579.

Bland R.C., Newman S.C. \& Orn H. (1988a). Period prevalence of psychiatric disorders in Edmonton. Acta Psychiatrica Scandinavica, Supplementum No. 338, vol. 77, pp. 95-120.

Bland R.C., Orn H. \& Newman S.C. (1988b). Lifetime prevalence of psychiatric disorders in Edmonton. Acta Psychiatrica Scandinavica, Supplementum No. 338, vol. 77, pp. 24-32.

Blazer D. \& Williams C.D. (1980). Epidemiology of dysforia and depression in an elderly population. American Journal of Psychiatry 137, 439-444.

Brown G.W. \& Harris T. (1978). The Social Origins of Depression. Tavistock Publications: London. 
Boyd J.H. \& Weissman M.M. (1981). Epidemiology of affective disorders. A reexamination and future directions. Archives of General Psychiatry 38, 1039-1046.

Boyd J.H., Burke J.D., Gruenberg E., Holzer C.E., Rae D.S., George L.K., Karno M., Stolzman R., McEvoy L. \& Nestadt G. (1984). Exclusion criteria of DSM-III. Archives of General Psychiatry 41, 983-989.

Breier A., Charney D.S. \& Heninger G.R. (1985). The diagnostic validity of anxiety disorders and their relationship to depressive illness. American Journal of Psychiatry 142, 787-797.

Burke K.C., Burke J.D., Rse D.S. \& Regier D.A. (1991). Comparing age at onset of major depression and other psychiatric disorders by birth cohorts in five US community populations. Archives of General Psychiatry 48, 789-795.

Cadoret R., Widmer B.B. \& Troughton E.P. (1980). Somatic complaints: Harbinger of depression in primary care. Journal of Affective Disorders 2, 61-70.

Canino G.J., Bird H.R., Shrout P.E., Rubio-Stipec M., Bravo M., Martinez R., Sesman M. \& Guevara L.M. (1987). The prevalence of specific psychiatric disorders in Puerto Rico. Archives of General Psychiatry 44, 725-735.

Costello C.G. (1982). Fears and phobias in women: a community study. Journal of Abnormal Psychology 91, 280-286.

Cross-National Collaborative Group (1992). The changing rate of major depression. Cross-national comparisons. Journal of American Medical Association 268, 3098-3105.

Dean C., Surtees P.G. \& Sashidaran S.P. (1983). Comparison of Research Diagnostic Systems in an Edinburgh community sample. British Journal of Psychiatry 142, 247-256.

Dew M.A., Bromet E.J., Schulberg H.C., Parkinson D.K. \& Curtis E.C. (1991). Factors affecting service atilization for depression in a white collar population. Social Psychiatry and Pychiatric Epidemiology 26, 230-237.

DiNardo P.A., O'Brien G.T. \& Barlow D.H. (1983). Reliability of DSM-III anxiety disorder categories using a new structured interview. Archives of General Psychiatry 40, 1070-1074.

Duncan-Jones P. \& Henderson S. (1978). The use of a two-phase design in a prevalence survey. Social Psychiatry 13, 231-237.

Eaton J.W. \& Weil R.J. (1955). Culture and Mental Disorders. Free Press: Glencoe, Illinois.

Essen-Moller E. \& Hagnell O. (1961). The frequency and risk of depression within a rural population group in Scania. Acta Psychiatrica Scandinavica, Supplementum No. 162, pp. 28-32.

Faravelli C., Guerrini degl'Innocenti B. \& Giardinelli L. (1989). Epidemiology of anxiety disorders in Florence. Acta Psychiatrica Scandinavica 79, 308-312.

Faravelli C., Guerrini Degl'Innocenti B., Aiazzi L., Incerpi G. \& Pallanti S. (1990). Epidemiology of mood disorders: a community survey in Florence. Journal of Affective Disorders 20, 135-141.

Goldberg D.P. \& Blackwell B. (1970). Psychiatric illness in general practice. A detailed study using a new method of case identification. British Medical Journal 1970, II, 439-443.

Hallstrom T. (1973). Mental disorder and sexuality in the climateric: a study in psychiatric epidemiology. In Reports From the Psychiatric Research Centre, St.. Joergen's Hospital, University of Goteborg, Sweden (ed. H. Forssman). Scandinavian University Books: Copenhagen.

Hecht H. \& Wittchen H.U. (1988). The frequency of social dysfunction in a general population sample and in patients with mental disorders. A comparison using the Social Interview Schedule (SIS). Social Psychiatry and Psychiatric Epidemio$\log y 23,17-29$.
Helgason T. (1961). Frequency of depressive states within geographically delimited population groups: the frequency of depressive states in Iceland as compared with the other Scandinavian countries. Acta Psychiatrica Scandinavica, Supplementum No. 162, pp. 81-90.

Hwu H.G., Yeh E.K. \& Chang L.Y. (1989). Prevalence of psychiatric disorders in Taiwan defined by the Chinese Diagnostic Interview Schedule. Acta Psychiatrica Scandinavica 79, 136147.

Karam E.G., Barakeh M., Karam A.N. \& El-Khouri N. (1991). The Arabic Diagnostic Interview Schedule. Revue Medical Libanaise 3, 28-30.

Karno M., Hough R.L., Burman M.A., Escobar J.I., Timbers D.M., Santana F. \& Boyd J.H. (1987). Lifetime prevalence of specific psychiatric disorders among Mexican Americans and non-hispanic whites in Los Angeles. Archives of General Psychiatry 44, 695-701.

Katon W., Kleinman A. \& Rosen G. (1982) Depression and somatization: a review. Part I. American Journal of Medicine 72, 127-135.

Kellner R. (1986). Somatization and Hypochondriasis. PraegerGreenwood: New York.

Kellner R. (1988). Anxiety and somatic complaints. In Handbook of Anxiety. II (ed. R. Noyes, M. Roth and G.D. Burrows). Elsevier : Amsterdam.

Kellner R., Simpson G.M. \& Winslow W.W. (1972). The relationship of depressive neurosis to anxiety and somatic symptoms. Psychosomatics 13, 358-362.

Klerman G.L. \& Weissman M.M. (1992). The course, morbidity, and costs of depression. Archives of General Psychiatry 49, 831-834.

Lemkau P.V., Tietze C. \& Cooper M. (1941). Mental hygiene problems in an urban district: I. Description of the study. Mental Hygiene 25, 624-646.

Lepine J.P., Lellouch J. \& Lovell A. (1989). Anxiety and depressive disorders in a French population: methodology and preliminary results. Psychiatry and Psychobiology 4, 267-274.

Lin T.-Y. (1953). A study of the incidence of mental disorder in Chinese and other culture. Psychiatry 16, 313.

Marks I. \& Lader M. (1973). Anxiety states (anxiety neurosis): a review. Journal of Nervous and Mental Diseases 156, 3-18.

Mazer M. (1967). Psychiatric disorders in general practice: the experience of an island community. American Journal of Psychiatry 124, 609-615.

Mintz J., Mintz L.I., Arruda M.J. \& Hwang S.S. (1992). Treatments of depression and the functional capacity to work. $A r$ chives of General Psychiatry 49, 761-768.

Murphy J.M. (1980). Continuities in community-based psychiatric epidemiology. Archives of General Psychiatry 37, 1215-1223.

Myers J.K., Weissman M.M., Tischler G.L., Holzer C.E., Leaf P.L., Orvaschel H., Anthony J.C., Boyd J.H., Burke J.D., Kramer M. \& Stoltzman R. (1984). Six month prevalence of psychiatric disorders in three communities. Archives of General Psychiatry 41, 959-967.

Nielsen J. \& Nielsen J.A. (1979). Treatment prevalence in a community mental health service with special regard to depressive disorders. Comprehensive Psychiatry 20, 67-77.

Regier D.A., Myers J.K., Kramer M., Robins L.N., Blazer D.G., Hough R.L., Eaton W.W. \& Locke B.Z. (1984). The NIMH Epidemiologic Catchment Area Program. Archives of General Psychiatry 41, 934-941.

Regier D.A., Boyd J.H., Burke J.D., Rae D.S., Myers J.K., Kramer M., Robins L.N., George L.K., Karno M. \& Locke B.Z.

Epidemiologia e Psichiatria Sociale, 3, 1, 1994 
(1988). One-month prevalence of mental disorders in the United States. Archives of General Psychiatry 45, 977-986.

Regier D.A., Narrow W.E. \& Rae D.S. (1990). The epidemiology of anxiety disorders: The Epidemiologic Catchment Area (ECA) experience. Journal of Psychiatric Research 24 (Suppl. 2), 3-14.

Robins L.N., Helzer J.E., Weissman M.M., Orvaschel H., Gruenberg E., Burke J.D. \& Regier D.A. (1984). Lifetime prevalence of specific psychiatric disorders in three sites. $A r$ chives of General Psychiatry 41, 949-958.

Schwartz M., Blazer D., George L. \& Landerman R. (1986). Somatization disorder in a community population. American Journal of Psychiatry 143, 1403-1408.

Shapiro S., Skinner E.A., Kessler L.G., VonKorff M., German P.S., Tischler G.L., Leaf P.J., Benham L., Cottler L. \& Regier D.A. (1984). Utilization of health and mental health services. Archives of General Psychiatry 41, 971-978.

Shepherd M., Cooper A.C., Brown A.C. \& Kalton G.W. (1966). Psychiatric Illness in General Practice. Oxford University Press:London.

Skuse D. \& Williams P. (1984). Screening for psychiatric disorder in general practice. Psychological Medicine 14, 365-377.

Sorensen A. \& Stroemgren E. (1961). Frequency of depressive states within geographically delimited population groups: prevalence (the Samso investigation). Acta Psychiatrica Scandinavi$\mathrm{ca}$, Supplementum No. 162, pp. 62-68.

Stoudemire A., Frank R. \& Hedemark N. (1986). The economic burden of depression. General Hospital Psychiatry 8, 387-394.

Thacore U.R., Gupta S.C. \& Suraiya M. (1975). Psychiatric morbidity in a north Indian community. British Journal of Psychiatry 126, 364-369.

Tyrer P. (1976). The Role of Bodily Feelings in Anxiety. Oxford University Press: London.

Uhlenhuth E.D., Baiter .B., Mellinger G.D. Cisin I.H. \& Clinthorne J. (1983). Symptom checklist syndromes in the general population: correlations with psychotheratepeutic drug use. Archives of General Psychiatry 40, 1167-1173.

Watts C.A.H., Conte E.C. \& Kuenssberg E.V. (1964). Survey of mental illness in general practice. British Medical Journal 2, 1351-1359.

Weissman M.M. \& Myers J.K. (1978). Affective disorders in a US urban community: the use of Research Diagnostic Criteria in an epidemiological survey. Archives of General Psychiatry 35, 1304-1311.
Weissman M.M., Myers J.K. \& Harding P.S. (1978). Psychiatric disorders in a U.S. urban community: 1975-1976. American Journal of Psychiatry 135, 459-462.

Weissman M.M., Leaf P.J., Tischler G.L., Blazer D.G., Karno M., Livingstone B. M. \& Florio L.P. (1988). Affective disorders in five United States communities. Psychological Medicine 18, 141-153.

Wells J.E., Bushnell J.A., Hornblow A.R., Joyce P.R. \& OakleyBrowne M.A. (1989). Christchurch Psychiatric Epidemiology Study, part I: methodology and lifetime prevalence for specific psychiatric disorders. Australian and New Zealand Journal of Psychiatry 23, 315-326.

Wing L., Wing J.K. \& Hailey A. (1967). The use of psychiatric services in three urban areas: a international case register study. Social Psychiatry 2, 158-167.

Wing J.K., Mann S.A. \& Leff J.P. (1978). The concept of a «case" in psychiatric population surveys. Psychological Medicine 8, 203-217.

Wittchen H.U. (1986). Epidemiology of panic attacks and panic disorders. In Panic and Phobia (ed. I. Hand and H.U. Wittchen), pp. 18-28.

Wittchen H.U. (1988). Natural course and spontaneous remission of untreated anxiety disorders - results of the Munich Followup Study (MFS). In Panic and Phobias. II. Treatments and Variables Affecting Course and Outcome (ed. I. Hand and H.U. Wittchen). Springer: Heidelberg.

Wittchen H.U. \& von Zerssen D. (1988). Verlaufe behandelter und unbehandelter Depressionen and Angststoerungen - Eine klinish-psychiatrische und epidemiologische Verlaufuntersuchung. Springer: Berlin.

Wittchen H.U. \& Essau C.A. (1989). Comorbidity of anxiety disorders and depression: does it affect course and outcome? Psychiatry and Psychobiology 4, 315-323.

Wittchen H.U. \& Essau C.A. (1993). Comorbidity and mixed anxiety-depressive disorders: is there epidemiologic evidence? Journal of Clinical Psychiatry 54 (suppl. 1), 9-15.

Wittchen H.U., Essau C.A., von Zerrsen D., Krieg J.C. \& Zaudig M. (1992). Lifetime and six-month prevalence of mental disorders in the Munich follow-up study. European Archives of Psychiatry and Clinical Neurosciences 241, 247-258 .

Yoo P.S. (1961). Mental disorders in Corean and rural communities. In Proceedings of the III World Congress of Psychiatry, pp. 1305-1369. Montreal. 